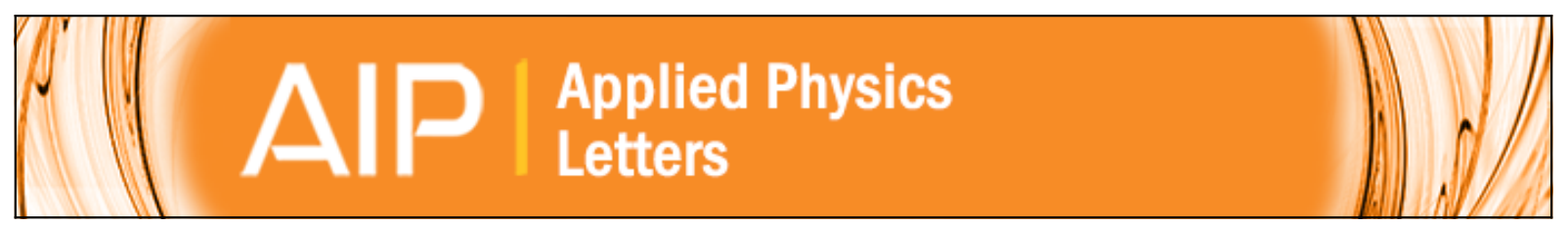

\title{
Suppression of the antiferroelectric phase during polarization cycling of an induced ferroelectric phase
}

Xiaoming Liu and Xiaoli Tan

Citation: Applied Physics Letters 107, 072908 (2015); doi: 10.1063/1.4929322

View online: http://dx.doi.org/10.1063/1.4929322

View Table of Contents: http://scitation.aip.org/content/aip/journal/apl/107/7?ver=pdfcov

Published by the AIP Publishing

\section{Articles you may be interested in}

The influence of temperature induced phase transition on the energy storage density of anti-ferroelectric ceramics

J. Appl. Phys. 118, 124107 (2015); 10.1063/1.4931886

Anomalous polarization in the antiferroelectric-ferroelectric phase coexistence state in $\mathrm{PbZrO} 3-\mathrm{Bi}(\mathrm{Mg} 1 / 2 \mathrm{Ti} 1 / 2) \mathrm{O} 3$ J. Appl. Phys. 114, 234105 (2013); 10.1063/1.4847475

Enhance of ferroelectric properties by modifying $\mathrm{Pb} 2+$ side by $\mathrm{Mg} 2+$ in PZT (52/48) ceramics

AIP Conf. Proc. 1512, 1276 (2013); 10.1063/1.4791518

Electric-field-induced and spontaneous relaxor-ferroelectric phase transitions in (Na1/2Bi1/2)1-xBaxTiO3

J. Appl. Phys. 112, 124106 (2012); 10.1063/1.4770326

Phenomenological theory of electric-field-induced phase transition behavior of antiferroelectric ceramic $(\mathrm{Pb}, \mathrm{Ba}, \mathrm{La})(\mathrm{Zr}, \mathrm{Sn}, \mathrm{Ti}) \mathrm{O} 3$ under uniaxial compressive pre-stress

J. Appl. Phys. 112, 034112 (2012); 10.1063/1.4744010

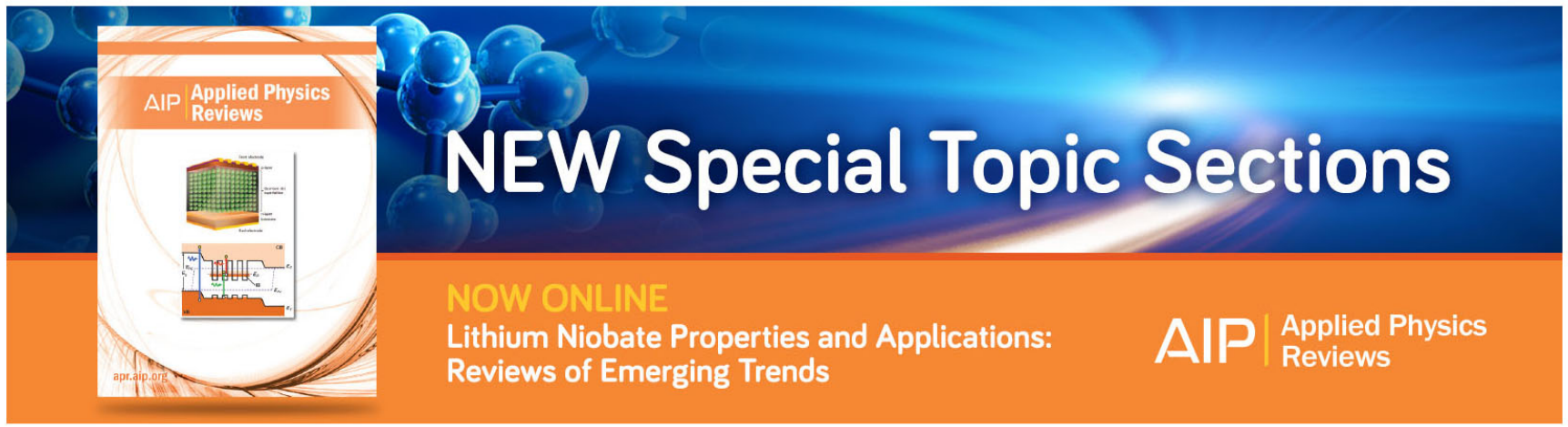




\title{
Suppression of the antiferroelectric phase during polarization cycling of an induced ferroelectric phase
}

\author{
Xiaoming Liu and Xiaoli Tan ${ }^{\text {a) }}$ \\ Department of Materials Science and Engineering, Iowa State University, Ames, Iowa 50011, USA
}

(Received 22 June 2015; accepted 8 August 2015; published online 21 August 2015)

\begin{abstract}
The ceramic $\mathrm{Pb}_{0.99} \mathrm{Nb}_{0.02}\left[\left(\mathrm{Zr}_{0.57} \mathrm{Sn}_{0.43}\right)_{0.92} \mathrm{Ti}_{0.08}\right]_{0.98} \mathrm{O}_{3}$ can exist in either an antiferroelectric or a ferroelectric phase at room temperature, depending on the thermal and electrical history. The antiferroelectric phase can be partially recovered from the induced ferroelectric phase when the applied field reverses polarity. Therefore, polarization cycling of the ferroelectric phase in the ceramic under bipolar fields at room temperature is accompanied with repeated phase transitions. In this letter, the stability of the recovered antiferroelectric phase upon electrical cycling of the ceramic is investigated. Ex-situ X-ray diffraction reveals that bipolar cycling suppresses the antiferroelectric phase; this is indirectly supported by piezoelectric coefficient $d_{33}$ measurements. It is speculated that the accumulated charged point defects during polarization cycling stabilize the polar ferroelectric phase. The findings presented are important to the fundamental studies of electric fatigue and field-induced phase transitions in ferroelectrics. (C) 2015 AIP Publishing LLC.

[http://dx.doi.org/10.1063/1.4929322]
\end{abstract}

An antiferroelectric (AFE) crystal contains antiparallel electric dipoles in its unit cell ${ }^{1}$ and experiences an electric field-induced transition to a ferroelectric (FE) phase if the critical field $\left(E_{\mathrm{F}}\right)$ is below the dielectric breakdown strength. ${ }^{2}$ In $\mathrm{PbZrO}_{3}$-based AFE polycrystalline ceramics, the most studied AFE oxide, the large increase in polarization at this phase transition occurs with an abrupt volume expansion. ${ }^{2-7}$ Almost all the $\mathrm{PbZrO}_{3}$-based AFE compositions reported in the literature exhibit a reversible AFE-FE phase transition (i.e., the AFE phase fully reappears before the applied electric field reaches zero during unloading). This is the physical basis for many device applications including high energy density capacitors and large displacement actuators. 3,8

$\mathrm{Sn}, \mathrm{Ti}$, and $\mathrm{Nb}$ are quite commonly used chemical modifiers to tune the stability of the AFE and FE phases and hence to manipulate the AFE-FE phase transition in $\mathrm{PbZrO}_{3}$-based ceramics. ${ }^{4-7,9-12}$ For example, in the $\mathrm{Pb}_{0.99} \mathrm{Nb}_{0.02}\left[\left(\mathrm{Zr}_{0.57} \mathrm{Sn}_{0.43}\right)_{1-y} \mathrm{Ti}_{y}\right]_{0.98} \mathrm{O}_{3} \quad$ (abbreviated as PNZST 43/100 $y / 2$ ) composition series at room temperature ${ }^{13-17}$ the AFE phase is quite stable for $y \leq 0.06$ and is fully recovered during unloading of the applied electric field. The two competing phases are nearly equally stable in $0.07 \leq y \leq 0.08$ and the electric field-induced AFE-to-FE transition becomes irreversible. The induced FE phase remains and the AFE phase is not recovered upon removal of the applied field. With still higher Ti content, the asprocessed ceramics are $\mathrm{FE}$ at room temperature regardless of thermal history.

We recently revealed that AFE ceramics with an irreversible AFE-to-FE phase transition are unique in a sense that the AFE phase can be partially recovered by moderate electric fields with a reverse polarity. ${ }^{15,17,18}$ Such an electric field-induced FE-to-AFE phase transition is highly unusual

\footnotetext{
${ }^{\text {a) }}$ Author to whom correspondence should be addressed. Electronic mail: xtan@iastate.edu
}

since electric field is known to favor a polar FE phase over a nonpolar AFE phase. This phenomenon is understood in terms of the distinct kinetics against the competing polarization reversal of the induced FE phase. ${ }^{18}$ If such a ceramic is subject to cyclic bipolar fields, repeated phase transitions are therefore expected to take place, accompany with repeated polarization reversals which eventually result in electric fatigue. ${ }^{19-23}$ In this work, the impact of cyclic bipolar fields on the AFE-FE phase transition in the polycrystalline PNZST 43/8.0/2 ceramic was investigated with ex-situ X-ray diffraction.

High density PNZST 43/8.0/2 ceramic disks were fabricated using hot isostatic pressing. ${ }^{16}$ A standardized ferroelectric test system (RT-66 A, Radiant Technologies) was used to record the polarization $(P)$ hysteresis loops under bipolar electric fields with an amplitude of $30 \mathrm{kV} / \mathrm{cm}$ and a frequency of $4 \mathrm{~Hz}$ at room temperature. Longitudinal strain $\left(x_{33}\right)$ was monitored separately with an MTI-2000 Fotonic Sensor (MTI Instruments, Inc., Albany, NY) at the same field amplitude but at $0.05 \mathrm{~Hz}$. This frequency was selected to maximize the number of data points and enhance the measurement resolution. For the electric fatigue test, cyclic bipolar fields of $\pm 30 \mathrm{kV} / \mathrm{cm}$ at $2 \mathrm{~Hz}$ were applied to a disk specimen to minimize the possible temperature change during cycling. It should be made clear that the different frequencies used for different measurements here did not affect the conclusions drawn from the present study. The phase structure of the specimen was analyzed with X-ray diffraction (Model D500, Siemens, Germany) with a step size of $0.02^{\circ}$ and a collection time of 30 seconds per step. Prior to the X-ray diffraction test, diluted nitric acid was used to chemically remove the sputtered silver electrodes. The recovery of the AFE phase was also assessed through the change of the piezoelectric coefficient $\left(d_{33}\right)$ with a piezo- $d_{33}$ meter (Model ZJ-4B, Institute of Acousitcs, China).

According to our previous study, the phase sequence in PNZST 43/8.0/2 during cooling is paraelectric, AFE, and 
then FE. ${ }^{16,17}$ The AFE-FE phase transition occurs at $-8^{\circ} \mathrm{C}$ during cooling while at $43{ }^{\circ} \mathrm{C}$ during heating. The large thermal hysteresis, which indicates the first-order nature of the transition, makes it possible for the ceramic to exist either as AFE or as $\mathrm{FE}$ at room temperature $\left(25^{\circ} \mathrm{C}\right)$. The development of polarization $(P)$ and strain $\left(x_{33}\right)$ from a virgin AFE ceramic pellet under the first cycle of bipolar electric fields is displayed in Fig. 1. Both $P$ and $x_{33}$ exhibit a sudden jump at $\sim 12 \mathrm{kV} / \mathrm{cm}$, which is noted as the critical field $E_{\mathrm{F}}$ for the AFE-to-FE phase transition. At the peak field of $30 \mathrm{kV} / \mathrm{cm}, P$ reads $34.4 \mu \mathrm{C} / \mathrm{cm}^{2}$ and $x_{33}$ reads $0.22 \%$. Consistent with previous studies, ${ }^{16,17}$ the induced FE phase remains and the AFE phase is not recovered during unloading of the field in the second quarter cycle. The ceramic seems to behave like a classic FE with regards to its macroscopic polarization and strain during the 3 rd and 4 th quarter cycle of applied field. Compared to the first half cycle, the strain range is significantly reduced to $0.07 \%$, varying from $0.14 \%$ to $0.21 \%$. However, as suggested by our previous work, ${ }^{15,17}$ phase transitions (first FE-to-AFE and then AFEto-FE) are expected in the 3rd quarter cycle when the field reverses its polarity.

During further application of bipolar fields after the first cycle, full reversal of the polarization is noticed while the strain range remains around 0.07\%. As shown in Fig. 2(a), square-shaped polarization hysteresis loops stay almost unchanged up to 50000 cycles. The evolution of the polarization at peak field, $P_{\max }$, remanent polarization, $P_{\mathrm{r}}$, and coercive field, $E_{\mathrm{c}}$, are displayed in Fig. 2(b). It is evident that both $P_{\max }$ and $P_{\mathrm{r}}$ do not show any degradation until 20000 cycles. After 50000 cycles of bipolar fields of $\pm 30 \mathrm{kV} / \mathrm{cm}$, $P_{\mathrm{r}}$ experiences about $4 \%$ reduction. At the same time, $E_{\mathrm{c}}$ remains unchanged in our fatigue experiment. It should be pointed out that $E_{\mathrm{c}}$ becomes higher in most ferroelectric ceramics after polarization cycling. ${ }^{19-23}$ The invariance of $E_{\mathrm{c}}$ in PNZST 43/8.0/2 may be largely attributed to the phase transition during cycling. As will be shown in the following paragraphs, the phase transition diminishes after 50000 cycles. The ceramic is expected to behave like a normal ferroelectric beyond 50000 cycles, and hence, increases in $E_{\mathrm{c}}$ are expected.

Since the dimensions of the virgin ceramic pellet were not resumed after the very first cycle of applied field, the

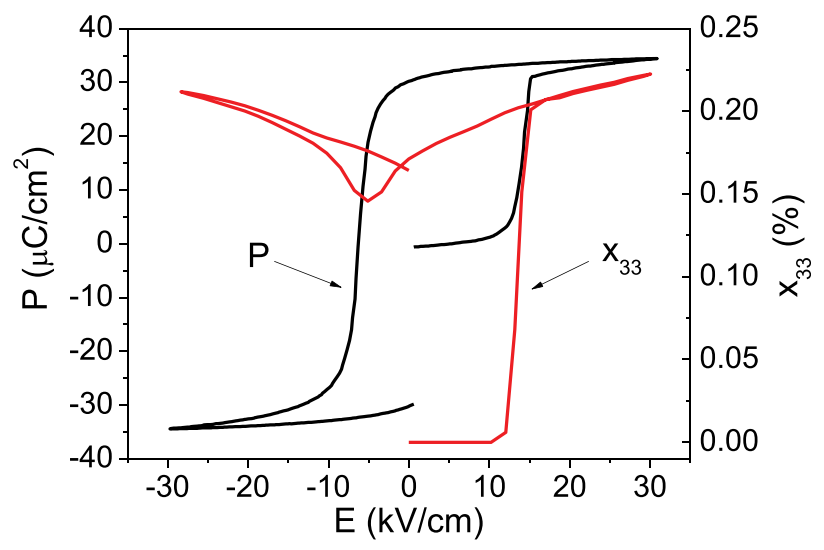

FIG. 1. The first cycle of the $P$ vs. $E$ and $x_{33}$ vs. $E$ hysteresis loops measured on a virgin PNZST 43/8.0/2 ceramic at room temperature.
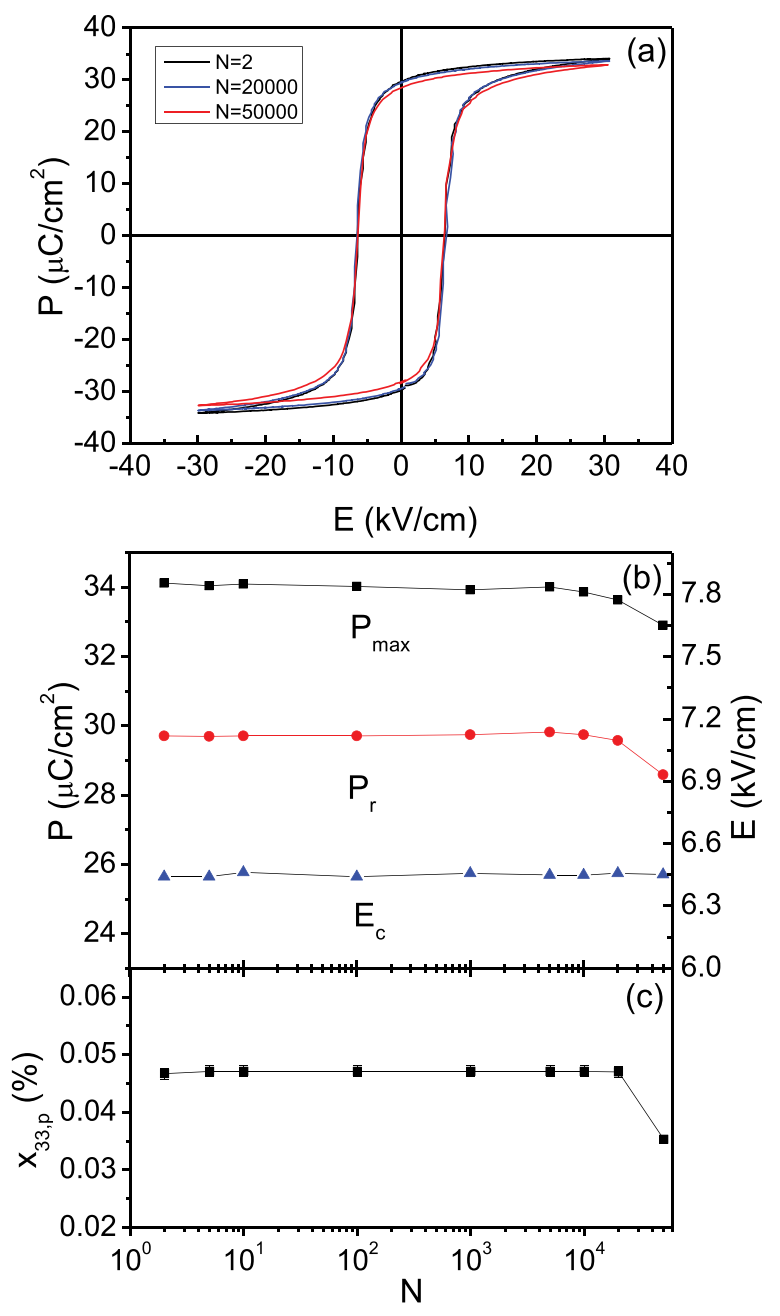

FIG. 2. Electric fatigue of the PNZST 43/8.0/2 ceramic under bipolar fields at a frequency of $2 \mathrm{~Hz}$ and room temperature. Evolution of (a) the polarization hysteresis loops, (b) the polarization at $30 \mathrm{kV} / \mathrm{cm}, P_{\max }$, the remanent polarization, $P_{\mathrm{r}}$, and the coercive field, $E_{\mathrm{c}}$, and (c) the strain at $30 \mathrm{kV} / \mathrm{cm}$, $x_{33, \mathrm{p}}$ against the cycle number, $N$. The strain $x_{33}$ is set to zero at zero field since the second cycle. Error bars are smaller than the size of the symbols.

zero point for $x_{33}$ was reset at zero applied field in subsequent electric cycles. The change of the strain at the peak field $(30 \mathrm{kV} / \mathrm{cm}), x_{33, \mathrm{p}}$, with respect to the cycle number, $N$, is plotted in Fig. 2(c). It appears that $x_{33, \mathrm{p}}$ correlates well with $P_{\max }$ and $P_{\mathrm{r}}$ (i.e., it is stable up to 20000 cycles and then decreases upon further cycling).

It was previously shown that $\mathrm{PbZrO}_{3}$-based antiferroelectric ceramics are characterized by the presence of incommensurate modulations, which disappear at the AFE-to-FE phase transition. ${ }^{5,7,9,10,17,24}$ The satellite peaks from the incommensurate modulation are also detectable with X-ray diffraction. As exemplified in Fig. 3(a), there are two satellite peaks adjacent to the fundamental peak $(210)_{c}$ in the AFE phase. It should be pointed out that the satellite peak at $2 \theta \approx 48.5^{\circ}$ overlaps with the $1 / 2(330)_{\mathrm{c}}$ superlattice peak and is hence stronger and sharper. This peak is then used as the signature of the AFE phase during electric cycling, shown in Fig. 3(b). It can be seen that after 1 full cycle of bipolar field of $\pm 30 \mathrm{kV} / \mathrm{cm}$, the satellite peak is significantly reduced in intensity, indicating the FE phase becomes dominant. As electric cycling proceeds, this satellite peak becomes weaker and almost completely disappears after 50000 cycles. The 


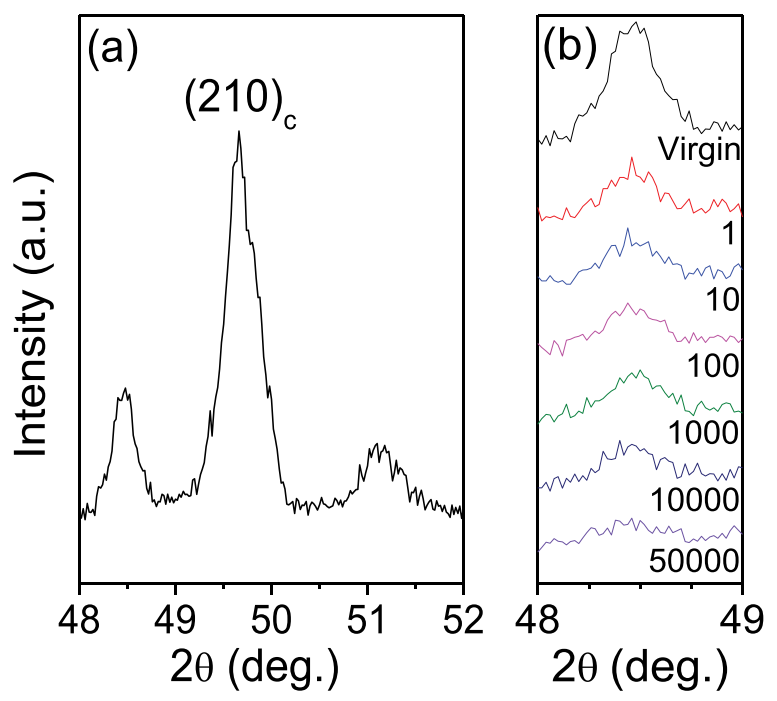

FIG. 3. The X-ray diffraction results for the PNZST 43/8.0/2 ceramic. (a) The $(210)_{\mathrm{c}}$ fundamental peak and the two adjacent satellite peaks in the virgin state, and (b) the satellite peak at $\sim 48.5^{\circ}$ after a series of electric cycles $(N)$.

vanishing of the satellite peak seems to suggest that bipolar electric cycling at room temperature suppresses the AFE phase and stabilizes the FE phase in PNZST 43/8.0/2.

The stabilizing effect is also verified by the recovery behavior of the AFE phase under reverse DC fields. Figure 4 displays the recovery process revealed by piezoelectric measurements and ex-situ X-ray diffraction on the satellite peak from a poled specimen and an electrically cycled specimen. As seen in Fig. 4(a), the satellite peak is initially strong in the virgin specimen and is flattened out after the poling process at room temperature. The specimen was then subject to a DC field of $3.8 \mathrm{kV} / \mathrm{cm}$ in the direction opposite to the poling field. It is evident that the satellite peak, the signature of the AFE phase, gradually reappears. Thus, the X-ray diffraction tests verify the electric field-induced FE-to-AFE phase transition on a bulk specimen and its slow kinetics. This is consistent with our recent in-situ transmission electron microscopy observations on individual grains in this ceramic. ${ }^{17}$

The recovery of the AFE phase from the poled specimen was also evaluated by the $d_{33}$ measurement. The AFE phase is non-polar and is not piezoelectric. The poling process induces the FE phase and aligns its polarization vectors, making the ceramic piezoelectric with the $d_{33} \sim 71 \mathrm{pC} / \mathrm{N}$. As displayed in Fig. 4(b), $d_{33}$ gradually decays to $27 \mathrm{pC} / \mathrm{N}$ under a reverse field of $3.8 \mathrm{kV} / \mathrm{cm}$ after $60 \mathrm{~min}$. It should be noted that the aligned polarizations in the induced FE phase may also randomize under the reverse field, leading to the reduction in $d_{33}$. However, the $\mathrm{X}$-ray diffraction results shown in Fig. 4(a) seem to suggest that the slow recovery of the nonpiezoelectric AFE phase under the reverse field is likely to be the main cause. Figure 4(b) also displays the evolution of $d_{33}$ under different reverse DC fields. As expected, a higher DC field leads to a faster decay of $d_{33}$. These data can be fitted well by the Kohlrausch-Williams-Watt (KWW)-type relation, ${ }^{25,26} d_{33}=d_{0}+d_{1} \exp \left[-\left(\frac{t}{\tau_{d}}\right)^{\beta}\right]$, where $t$ is time in second, $d_{0}, d_{1}, \beta$, and $\tau_{\mathrm{d}}$ are fitting parameters, which are listed in Table I. The KWW-type relation was found to
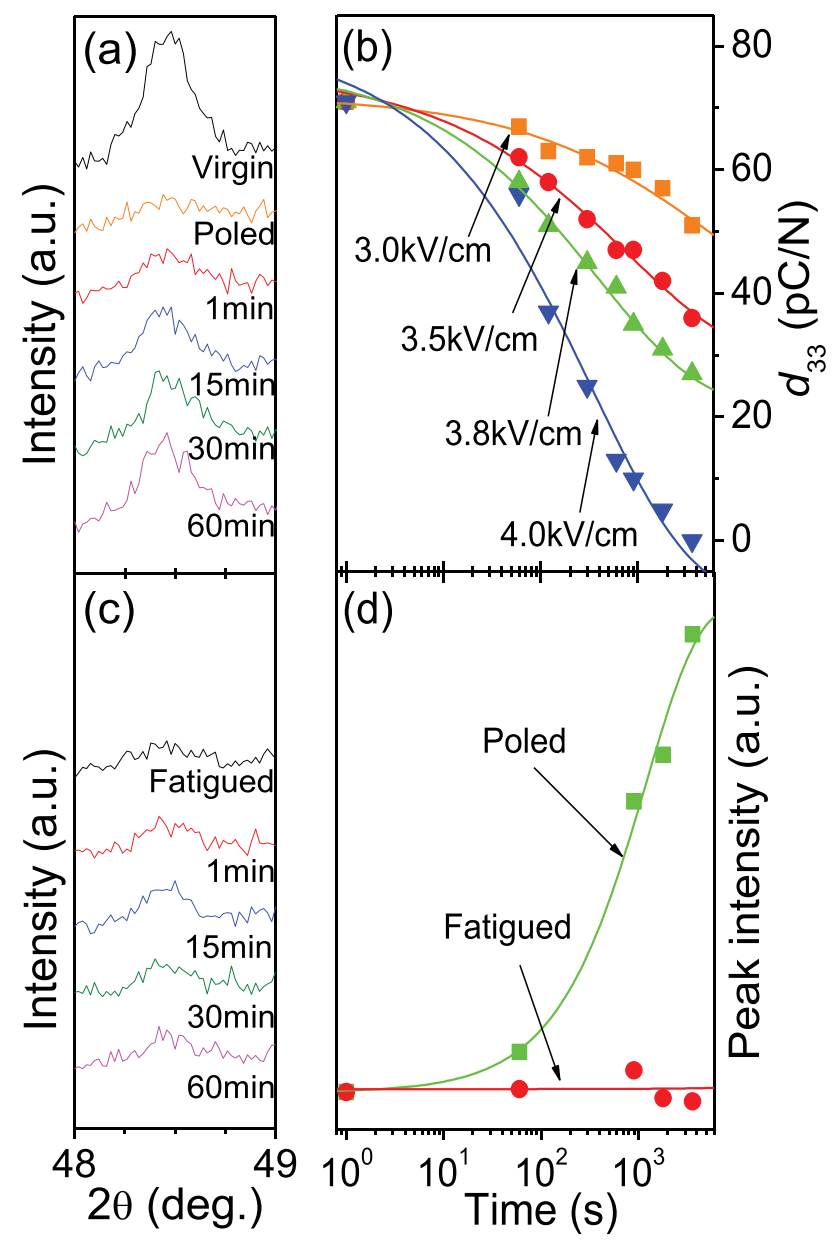

FIG. 4. (a) Evolution of the satellite peak at $\sim 48.5^{\circ}$ from the same surface of the same specimen at virgin and poled states, and after a series of time of application of a reverse DC field of $3.8 \mathrm{kV} / \mathrm{cm}$. The electric poling is conducted at $30 \mathrm{kV} / \mathrm{cm}$ for $10 \mathrm{~min}$ at room temperature. (b) The evolution of the piezoelectric coefficient $d_{33}$ of the same poled specimen under reverse DC fields of $3.0,3.5,3.8$, and $4.0 \mathrm{kV} / \mathrm{cm}$. The symbols are measured data points, and the solid lines are fitting curves according to the KWW relation. (c) The evolution of the satellite peak under the reverse DC field of $3.8 \mathrm{kV} / \mathrm{cm}$ of a fatigued specimen after 50000 cycles of $\pm 30 \mathrm{kV} / \mathrm{cm}$ bipolar fields. (d) The integrated intensity of the satellite peak shown in (a) and (c). The solid lines are fitting curves according to the KWW relation. Error bars in (b) and (d) are smaller than the size of the symbols.

describe well the flipping of polar nanoregions in the induced ferroelectric phase back to the initial disordered relaxor state due to the distribution of relaxation times. ${ }^{25-27}$ The exponent $\beta(0<\beta<1)$ indicates the width of this distribution; a smaller value implies a broader distribution. The parameter $\tau_{\mathrm{d}}$ represents the time constant for the process, ${ }^{27}$ which is $5500,800,400$, and 350 seconds for 3.0, 3.5, 3.8, and $4.0 \mathrm{kV} / \mathrm{cm}$ DC fields, respectively. These data suggest that the recovery of the nonpolar antiferroelectric phase from the induced ferroelectric phase is a slow process; the time

TABLE I. The fitting parameters for the evolution of $d_{33}$.

\begin{tabular}{lcccc}
\hline \hline $\mathrm{E}(\mathrm{kV} / \mathrm{cm})$ & $d_{0}(\mathrm{pC} / \mathrm{N})$ & $d_{1}(\mathrm{pC} / \mathrm{N})$ & $\tau_{\mathrm{d}}(\mathrm{s})$ & $\beta$ \\
\hline 3.0 & 37.0 & 35.0 & 5500 & 0.38 \\
3.5 & 29.0 & 47.0 & 800 & 0.38 \\
3.8 & 22.0 & 55.0 & 400 & 0.42 \\
4.0 & -9.0 & 90.0 & 350 & 0.43 \\
\hline \hline
\end{tabular}


constant is shorter with a narrower distribution at higher reverse fields.

However, the scenario just described is in sharp contrast to that of the electrically fatigued specimen, as shown in Fig. 4(c). In this case, the signature satellite peak remains extremely weak even after $60 \mathrm{~min}$ application of $3.8 \mathrm{kV} / \mathrm{cm}$. Evidently, polarization cycling of the induced ferroelectric phase has suppressed the recovery of the antiferroelectric phase under reverse DC fields.

The integrated intensities of the satellite peaks for the poled and the fatigued specimens under the reverse field of $3.8 \mathrm{kV} / \mathrm{cm}$ are quantitatively compared with respect to time in Fig. 4(d). Again, the data can be fitted with the KWWtype relation with $\tau_{\mathrm{d}}$ of 1200 and 140000 seconds for the poled and the fatigued conditions, respectively.

It should be noted again that the composition PNZST 43/8.0/2 at room temperature is at the AFE/FE phase boundary. ${ }^{13,14}$ Both the previous in-situ transmission electron microcopy study ${ }^{17}$ and the present $e x$-situ X-ray diffraction results verify that the AFE phase is partially recovered during the polarization reversal of the induced FE phase. Within one full cycle of applied bipolar fields, $180^{\circ}$ polarization reversal occurs twice and the $\mathrm{AFE} \leftrightarrow$ FE phase transition takes place four times, all of which happens in the first and the third quarter cycles. Even though they appear to compete against each other, the phase transition is a much slower process than polarization reversal. ${ }^{18}$ The ex-situ X-ray diffraction data presented in this letter indicate that repeated bipolar cycling shifts the balance in PNZST 43/8.0/2 in favor of the FE phase and corresponding polarization reversal and against the recovery of the AFE phase. It has long been known that electrical cycling on ferroelectrics leads to degradation in materials functionality due to various suggested mechanisms, most of which are associated with the accumulation of charged point defects. ${ }^{20-22}$ These charged defects create local electric fields and often form permanent dipoles. Therefore, it seems to be plausible that the charged point defects with increased amount in fatigued PNZST 43/8.0/2 favor the polar FE phase and suppress the non-polar AFE phase. The present work indicates that PNZST 43/8.0/2 is an ideal model material to the fundamental study of the complex electric fatigue phenomenon where charged point defects, polarization reversal, polar-nonpolar phase transition, and the associated mechanical and thermal effects are all involved. Additional characterization tools, including the in-situ transmission electron microcopy ${ }^{28}$ and the electron paramagnetic resonance technique ${ }^{29}$ will be needed to verify the accumulation of the charged point defects and the role they play during bipolar electrical cycling.

In summary, ex-situ X-ray diffraction tests on bulk PNZST 43/8.0/2 ceramic reveal the recovery of an AFE phase out of an induced FE phase during polarization reversal. Such an electric field-assisted FE-to-AFE phase transition displays much slower kinetics than polarization switching of the FE phase. Bipolar electrical cycling of the ceramic suppresses the recovery of the AFE phase. It is suggested that the accumulated charged point defects in electrically fatigued specimen stabilize the polar FE phase over the nonpolar AFE phase.

This work was supported by the National Science Foundation (NSF) of USA through Grant No. DMR1465254.

${ }^{1}$ C. Kittel, Phys. Rev. 82, 729 (1951).

${ }^{2}$ D. Berlincourt, H. H. A. Krueger, and B. Jaffe, J. Phys. Chem. Solids 25, 659 (1964).

${ }^{3}$ W. Y. Pan, C. Q. Dam, Q. M. Zhang, and L. E. Cross, J. Appl. Phys. 66, 6014 (1989).

${ }^{4}$ P. Yang and D. A. Payne, J. Appl. Phys. 71, 1361 (1992).

${ }^{5}$ D. Viehland, D. Forst, Z. Xu, and J. F. Li, J. Am. Ceram. Soc. 78, 2101 (1995).

${ }^{6}$ Z. Dai, Z. Xu, and X. Yao, Appl. Phys. Lett. 92, 072904 (2008).

${ }^{7}$ X. Tan, C. Ma, J. Frederick, S. Beckman, and K. G. Webber, J. Am. Ceram. Soc. 94, 4091 (2011).

${ }^{8}$ S. Kwon, W. Hackenberger, E. Alberta, E. Furman, and M. Lanagan, IEEE Electr. Insul. Mag. 27(2), 43 (2011).

${ }^{9} \mathrm{H}$. He and X. Tan, Appl. Phys. Lett. 85, 3187 (2004).

${ }^{10} \mathrm{H}$. He and X. Tan, Phys. Rev. B 72, 024102 (2005).

${ }^{11}$ X. Tan, W. Jo, T. Granzow, J. Frederick, E. Aulbach, and J. Rödel, Appl. Phys. Lett. 94, 042909 (2009).

${ }^{12}$ X. Tan, J. Frederick, C. Ma, E. Aulbach, M. Marsilius, and J. Rödel, Phys. Rev. B 81, 014103 (2010).

${ }^{13}$ H. He and X. Tan, J. Phys.: Condens. Matter 19, 136003 (2007).

${ }^{14}$ J. Frederick, X. Tan, and W. Jo, J. Am. Ceram. Soc. 94, 1149 (2011).

${ }^{15}$ X. Tan, J. Frederick, C. Ma, W. Jo, and J. Rödel, Phys. Rev. Lett. 105, 255702 (2010).

${ }^{16}$ X. Tan, S. E. Young, Y. H. Seo, J. Y. Zhang, W. Hong, and K. G. Webber, Acta Mater. 62, 114 (2014).

${ }^{17}$ H. Guo and X. Tan, Phys. Rev. B 91, 144104 (2015).

${ }^{18}$ Y. H. Xu, W. Hong, Y. J. Feng, and X. Tan, Appl. Phys. Lett. 104, 052903 (2014).

${ }^{19}$ K. Takemura, M. Ozgul, V. Bornand, S. Trolier-McKinstry, and C. A. Randall, J. Appl. Phys. 88, 7272 (2000).

${ }^{20}$ A. K. Tagantsev, I. Stolichnov, E. L. Colla, and N. Setter, J. Appl. Phys. 90, 1387 (2001)

${ }^{21}$ D. C. Lupascu and J. Rödel, Adv. Eng. Mater. 7, 882 (2005).

${ }^{22}$ X. J. Lou, J. Appl. Phys. 105, 024101 (2009).

${ }^{23}$ J. Glaum and M. Hoffman, J. Am. Ceram. Soc. 97, 665 (2014).

${ }^{24}$ Z. Xu, D. Viehland, P. Yang, and D. A. Payne, J. Appl. Phys. 74, 3406 (1993).

${ }^{25}$ V. Shvartsman, B. Dkhil, and A. Kholkin, Annu. Rev. Mater. Res. 43, 423 (2013).

${ }^{26}$ S. Kalinin, B. Rodriguez, J. Budai, S. Jesse, A. Morozovska, A. Bokov, and Z. G. Ye, Phys. Rev. B 81, 064107 (2010).

${ }^{27}$ A. Kumar, Y. Ehara, A. Wada, H. Funakubo, F. Griggio, S. TrolierMcKinstry, S. Jesse, and S. V. Kalinin, J. Appl. Phys. 112, 052021 (2012).

${ }^{28}$ H. Z. Guo, X. M. Liu, J. Rödel, and X. Tan, Adv. Funct. Mater. 25, 270 (2015).

${ }^{29}$ N. Raengthon, V. J. DeRose, G. L. Brennecka, and D. P. Cann, Appl. Phys. Lett. 101, 112904 (2012). 